\title{
Dimensional interaction of hue and brightness in preattentive field segregation
}

\author{
TARA C. CALLAGHAN \\ Yale University, New Haven, Connecticut
}

\begin{abstract}
The interaction of hue and brightness dimensions during preattentive processing was assessed using a novel task. The method combined the texture segregation task of Beck (1966) with the stimulus manipulations of Garner and Felfoldy (1970). Observers were required to make field segregation judgments for textured visual arrays. Fields were segregated by differences on a single dimension, either hue or brightness. For some arrays the second dimension had a constant level, for others it varied irrelevantly. In Experiment 1, the extent of interaction between hue and brightness dimensions was assessed at two levels of hue discriminability. Irrelevant variation of brightness interfered with segregation judgments based on hue at both levels of hue discriminability. Hue variation did not interfere with judgments based on brightness at either level. Segregation judgments were longer for both hue and brightness arrays in the hard than in the easy hue discriminability stimulus set. In Experiment 2, all arrays were randomly mixed in an unblocked design to determine whether blocking in Experiment 1 produced a general slowing of response for arrays in the hard discriminability set. An identical pattern of asymmetric interference was revealed. Irrelevant variation of brightness interfered with hue segregation judgments at both discriminability levels. Hue variation did not interfere with judgments based on brightness at either level. In the unblocked design, only hue arrays were influenced by the hue discriminability manipulation. In Experiment 3, discriminability was reduced for brightness and enhanced for hue. A symmetric pattern, whereby hue variation interfered with brightness judgments and brightness variation with hue judgments, was obtained when hue was easy to discriminate. When hue was difficult to discriminate, only brightness variation interfered with hue judgments. These findings are consistent with the claim that simple properties of the stimulus mediate preattentive boundary judgments (Beck, 1966, 1982; Treisman, 1982). Furthermore, the results show that although the dimensions of hue and brightness can promote field segregation, they are highly susceptible to interference from other dimensions present in the array.
\end{abstract}

The segregation of textured visual displays into separate fields is a fundamental task of the human perceiver. Field segregation has been studied extensively by Beck (1966, 1967, 1972, 1982; Beck, Pradzny, \& Rosenfeld, 1983). A number of paradigms were employed in his experiments. In one (e.g., Beck, 1966), observers were required to indicate which section in a three-section array segregated most strongly from the other two. In a second (e.g., Beck, 1972), observers were presented with multielement arrays and asked to rate, on a scale of 1 to 100 , how well disparate elements segregated from the background.

A portion of this research was conducted while the author was a doctoral candidate at Brown University. The remainder was completed at Yale University, where the author is a postdoctoral fellow. The research was supported by a Natural Sciences and Engineering Research Council of Canada postdoctoral fellowship to Tara C. Callaghan, by National Institute of Mental Health Grant MH 14229 to Wendell R. Garner, and by National Institute of Child Health and Human Development Grant HD13406 to Bryan E. Shepp. The author is grateful to W. R. Garner and B. E. Shepp for their valuable comments on an earlier draft of this paper. Requests for reprints should be sent to Tara $C$. Callaghan, Department of Psychology, Box 11A, Yale Station, New Haven, CT 06520.
In a third task (e.g., Beck, 1972), the time required for observers to count disparate elements in these multielement arrays was measured.

Treisman (1982; Treisman \& Gelade, 1980) has also explored field segregation effects. In her procedure, observers are required to sort decks of cards into two groups. Each card contains a single array. Arrays differ according to whether fields segregate along either a horizontal or a vertical axis. There are two types of decks. In one, array fields are distinguished by differences in simple properties (e.g., color, shape). In the other, they are differentiated by differences in conjunctions of simple properties (e.g., color plus shape). Mean reaction time (RT) to sort different types of decks is measured.

Field segregation is produced by simple property differences between elements in the textured array (Beck, 1966, 1967, 1972, 1982; Treisman \& Gelade, 1980), and not by differences in complex (e.g., conjunctions of simple properties) properties (Beck, 1982; Treisman \& Gelade, 1980). In the present research, the question of whether these simple properties interact during field segregation was explored. Although the extent of dimensional interaction had 
been explored in studies that required the observer to directly attend to dimensions (e.g., Garner, 1974), it had not been investigated in field segregation, a process generally considered to occur prior to the focusing of attention (Beck, 1982; Kahneman, 1973; Treisman, 1982).

\section{Task Definitions: Preattentive vs. Attentive}

The distinction between the concepts of preattentional and attentional processing can be made clear with operational task definitions. In the field segregation task employed in the present experiments, as in the one employed by Treisman and Gelade (1980), observers are required to respond according to the orientation of fields (i.e., horizontal or vertical) in the stimulus array. There is no instruction to attend to the stimulus properties that define the fields. Thus, in order to perform this task, the observer need only attend to the overall orientation of fields, and not to the particular dimensional differences that define those fields. In contrast, for the speeded sorting task employed by Garner and his colleagues (e.g., Garner \& Felfoldy, 1970), the observer is specifically instructed to make a classification response based on a particular dimensional property of the stimulus. This response must be made in a situation in which other dimensional properties potentially vary. Therefore, in order to successfully perform the speeded sorting task, the observer must directly process dimensions of the stimulus.

For present purposes, we considered the field segregation task to be preattentive because the response to determine the orientation of fields is removed from direct processing of dimensions. Likewise, we considered the speeded sorting task as attentional because it compels observers to process dimensions in a direct manner.

The nature of processing for multidimensional stimuli has been extensively studied by Garner and his colleagues (Garner, 1970, 1974, 1976, 1978, 1983; Garner \& Felfoldy, 1970) in experiments that employed the second type of task (i.e., attentional). These researchers propose that the extent to which stimulus analysis is based on separate components or dimensions, as compared with overall similarity properties, is dependent both on the nature of the stimulus dimensions and on the demands of the task. This contention is similar to the claim (Kahneman, 1973; Neisser, 1967; Treisman, in press) that the nature of perceptual processing at preattentive levels of processing is potentially different from that at attentive levels of processing.

\section{Levels of Processing: Preattentive vs. Attentive}

Neisser (1967) distinguished between preattentive and attentive levels of processing in perception. This distinction was based on an assumption that preattention is an early, automatic stage of processing, the purpose of which is to define groups that are later subjected to more extensive, focused processing. The perception of fields in a textured multielement array and the segregation of objects from their backgrounds are examples of preattentive grouping (Beck, 1982; Treisman, 1982). Before attention can be directed to a particular field or object, that field or object must be distinguished from the background. Fields can be distinguished before the basis for the segregation (i.e., particular dimensional differences that define the fields) is perceived.

Another assumption that underlies the preattentive/ attentive distinction (Neisser, 1967) is that the nature of the analysis for visual stimuli (i.e., whether based on wholes or components) at the early (preattentive) stages of processing is potentially different from that at the later (attentive) stages of processing (Kahneman, 1973; Neisser, 1967; Treisman, 1979, in press). In this view, as in the Garnerian view, the manner in which the stimulus is processed will depend on the particular type of task.

Garner (1983) has provided insight into the importance of task factors with a comparison of speeded and nonspeeded classification tasks. In general, the requirement for a speeded response typically enhances the degree of dimensional interaction obtained (Garner, 1983). In addition to task factors, Garner (e.g., 1974) argues that stimulus properties are crucial. He proposes that the nature of multidimensional stimuli can be described by a continuum of dimensional interaction. At one extreme lie dimensions that show strong, mandatory interaction when combined (i.e., integral), and at the other extreme lie dimensions that retain their identity when combined in a stimulus (i.e., separable).

For a broader apprehension of the role of stimulus and task factors in perceptual processing, it is necessary to extend these investigations beyond the attentional task situation. This was accomplished in the present experiments, in which dimensional interaction was examined using a field segregation task. The stimuli employed were formed from hue and brightness dimensions, which have been found to show strong, mandatory interaction in attentional tasks (e.g., Garner, 1974; Shepp, 1983).

\section{Dimensional Interaction with Color Stimuli}

Stimuli formed from any pairwise combination of the attributes of color (i.e., hue, brightness, and saturation) consistently show strong dimensional interaction effects. Phenomenologically, these stimuli are seen as fused entities. Empirically, responses to colored stimuli in similarity scaling studies (Burns, Shepp, McDonough, \& Erlich, 1978; Handel \& Imai, 1972; Hyman \& Well, 1967; Torgerson, 1958) and restricted classification experiments (Handel \& Imai, 1972; Shepp, 1983) are based on overall similarity relations rather than dimensional structure. 
For present purposes, it is important to note the pattern that emerges for color stimuli in speeded classification tasks. Here, a failure of selective attention to component dimensions is revealed by interference in RT when dimensions are varied orthogonally and redundancy gains in $\mathrm{RT}$ when they are correlated. This pattern has been demonstrated using a variety of speeded tasks, including the original speeded sorting procedure, which provided a model for the preattentive task employed here (Garner \& Felfoldy, 1970; Shepp \& Swartz, 1976). Alternate versions that use selective attention and biconditional classification tasks (Gottwald \& Garner, 1975) or discrete trial priming tasks (Shepp, 1983) also produce this integral pattern of orthogonal interference and redundancy gain for color stimuli. The results from these studies unequivocally indicate that human perceivers cannot selectively attend to the separate attributes of color.

\section{Purpose of the Present Research}

In the present experiment we explored the nature of preattentive processing for multidimensional stimuli. The particular question that was addressed was whether or not mandatory dimensional interaction existed for color stimuli at a preattentive level of processing. In line with Beck (1982), it was proposed that field segregation is a fundamental, and early, task of the human perceiver. Beck has argued that segregation is dependent on the similarity relations between simple properties in a visual array. Thus, the elements are assumed to group together because they share a simple property. A prediction derived from this model is that strong segregation will occur when the properties of elements have high similarity within a region and low similarity between regions. Garner (1974) has argued that similarity relations are also important in attentional processing, especially for integral stimuli (e.g., those formed from the combination of the attributes of color). The present experiments attempted to form a bridge between preattentive and attentive levels of processing via an exploration of stimulus interaction effects in preattentive processing.

The preattentive field segregation task employed here combined the methods of Beck (1966) and Treisman and Gelade (1980) with the stimulus manipulations of Garner and Felfoldy (1970). The visual arrays for this task were segregated into two fields of equal size. The fields were oriented in either a horizontal or a vertical manner. The observer's task was to indicate which orientation was viewed.

The arrays were constructed so that orthogonal interference and redundancy gain effects, typically found for these stimuli in attentional tasks (Garner \& Felfoldy, 1970), could be assessed using a preattentive field segregation procedure. It was expected that the strong, mandatory interaction that characterizes hue and brightness dimensions during atten- tional processing (Garner, 1974; Shepp, 1983) would also be evident during preattentive processing.

\section{EXPERIMENT 1}

\section{Method}

\section{Experimental Conditions}

The general procedure employed in this experiment involved a series of presentations of single arrays composed of a square of 36 elements, each presentation lasting $200 \mathrm{msec}$. Observers were required to indicate, by pressing a response button, whether the two fields contained in the array were oriented in a horizontal or a vertical manner. A hypothetical array, with a horizontal field orientation, is illustrated in Figure 1.

Two experimental variables were manipulated in this experiment: Type of stimulus array and discriminability of the hue dimension.

Type of stimulus array. There were three types of stimulus arrays: Control, correlated, and orthogonal. The control and orthogonal array types were further subdivided according to the dimension (i.e., hue or brightness) that defined the fields. Figure 2 presents schematic representations for all types of stimulus arrays employed, with, for ease of illustration, 16-element arrays rather than the 36-element arrays that were actually used.

In control arrays, all 36 elements shared either a level of brightness (e.g., B1 for control-hue, as in Figure 2a) or hue (e.g., $\mathrm{Hl}$ for control-brightness, as in Figure $2 b$ ). When hue differences defined the fields (i.e., control-hue), the elements differed on a level of hue (e.g., $\mathrm{H} 1$ vs. $\mathrm{H} 2$ in Figure $2 \mathrm{a}$ ) across fields. When brightness differences established segregation (i.e., controlbrightness), elements differed on a level of brightness (e.g., B1 vs. B2 in Figure 2b) across fields. Thus, for control arrays, the 18 elements within a field shared a dimensional level and differed from elements in the second field by a level on that dimension, either hue (control-hue) or brightness (control-brightness).

In correlated arrays, dimensions were redundant since either hue or brightness differences could define the fields. Thus, the particular levels of dimension shared by elements in one field (e.g., H1B1 in Figure 2c) were different from those levels shared by

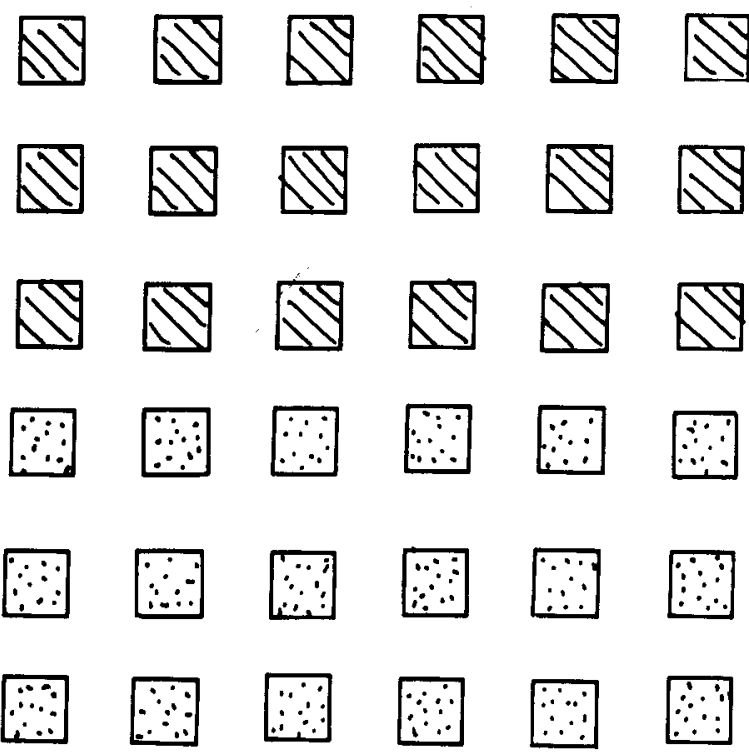

Figure 1. A hypothetical textured array that segregates into two fields along a horizontal axis. A vertical field orientation (not shown) can be observed by rotating this diagram 90 deg. 

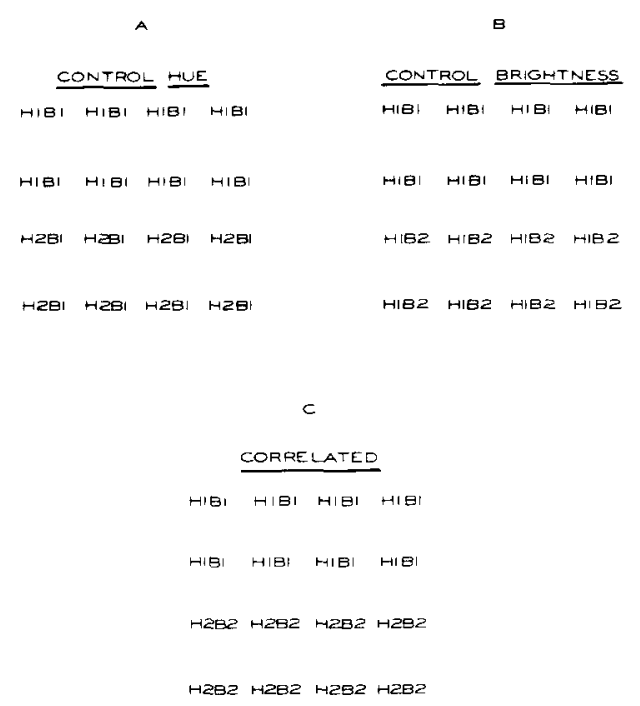

D.
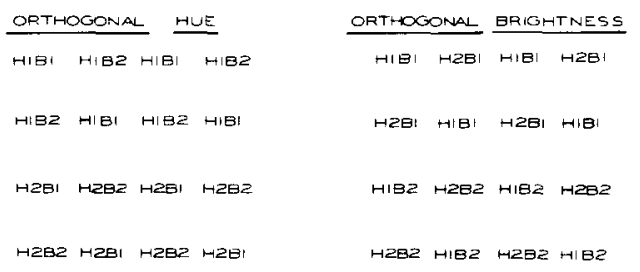

Figure 2. A schematic representation of the arrangement of elements for each subtype of army (control-hue, control-brightness, correlated, orthogonal-hue, orthogonal-brightness) used in the reaction time field segregation task. Letters denote a particular dimension ( $\mathrm{H}=$ hue, $\mathbf{B}=$ brightness), and numbers indicate the specific level on the dimension (see text for a detailed explanation).

elements in the second field (e.g., H2B2 in Figure 2c). In correlated arrays, the dissimilarity between fields was maximized by this redundant combination of dimensions. In speeded sorting tasks (e.g., Garner \& Felfoldy, 1970), redundant integral dimensions are typically accompanied by performance gains when compared with the faster of the two control conditions (redundancy effect). If this pattern were to hold for preattentive field segregation, then mean RT for correlated arrays should be faster than the faster mean RT of the two subtypes of control arrays.

In orthogonal arrays, the $\mathbf{1 8}$ elements within a field differed from those in the second field on a level of either hue (e.g., H1 vs. $\mathrm{H} 2$ for orthogonal-hue, as in Figure 2d) or brightness (e.g., B1 vs. B2 for orthogonal-brightness as in Figure 2e). For these arrays, the level on the second dimension alternated irrelevantly across all 36 elements. Thus, for orthogonal-hue arrays, fields were defined by a difference in hue and elements differed irrelevantly on the brightness dimension (e.g., B1/B2 in Figure 2d). For orthogonal-brightness arrays, fields were defined by a difference in brightness and the level of hue alternated irrelevantly across the entire array (e.g., $\mathrm{H} 1 / \mathrm{H} 2$ in Figure 2e). If segregation responses were not to be influenced by the irrelevant variation of levels on the second dimension for orthogonal arrays, then fields should segregate with as much ease as in the control arrays. On the other hand, if irrelevant variation of the second dimension were to interfere with segregation judgments, then mean RT should be longer for orthogonal arrays than for control arrays (interference effect).
Discriminability of the hue dimension. The discriminability between levels on the hue dimension was the second variable that was manipulated in this experiment. There were two stimulus sets, one for each level of hue discriminability. In the easy discriminability set, two levels of hue, separated by four steps in the Munsell color system ( $\mathrm{H1}=5 \mathrm{R} ; \mathrm{H} 2=5 \mathrm{RP}$ ), and two levels of brightness (value in the Munsell system), separated by two steps $(B 1=3$; $B 2=5$, were used in construction of the arrays. For arrays in the hard discriminability set, levels of hue $(\mathrm{H1}=5 \mathrm{R} ; \mathrm{H} 2=10 \mathrm{RP})$ and brightness $(B 1=3 ; B 2=5)$ were separated by two steps in the Munsell system.

Note that the discriminability manipulation was a variation on the hue dimension only. The levels of brightness were constant across stimulus sets (i.e., $B 1=3 ; B 2=5$ ), as was the chroma level (8 for all elements).

The hue discriminability factor was included in order to assess whether the pattern of dimensional interaction in a preattentive task was influenced by discriminability of component dimensions. Garner and Felfoldy (1970, Experiment 5) report less interference for a highly discriminable set of stimuli than for a set composed of less discriminable stimuli when they varied discriminability of brightness and saturation in a speeded sorting task. If this effect existed with the present task, then interference (i.e., longer mean RT for orthogonal than for control arrays) should be greater for arrays in the hard discriminability set than for those in the easy discriminability set.

\section{Subjects}

Sixteen Yale undergraduates were paid for their participation in this experiment. All had normal or corrected vision. Each observer participated in only one experimental session, which lasted approximately $1 \mathrm{~h}$.

\section{Apparatus}

The stimuli were $6 \times 6$ matrices (i.e., 36-element arrays) composed of square elements that varied in hue and brightness. Variation of the second dimension in the orthogonal arrays was accomplished by alternating the levels on that dimension across the array. Slides of these stimuli were prepared for use in a projection tachistoscope. The visual angle of the entire projected array was approximately $5.7 \mathrm{deg}$. Each of the elements subtended a visual angle of approximately $0.5 \mathrm{deg}$. The 36 elements were evenly arranged on a black surround. The spaces between the elements were approximately $0.54 \mathrm{deg}$ of visual angle.

The stimulus slides were presented in a Gerbrands randomaccess projection tachistoscope (Model G1177). The slides were rear-projected onto a screen that was mounted in a wooden display case. Approximate viewing distance was $50 \mathrm{~cm}$. The display case contained two response buttons, labeled $\mathrm{H}$ or $\mathrm{V}$, that activated a digital timer (Gerbrands Model G1271) when pressed.

\section{Experimental Design}

The observers were told that they would be viewing a series of multielement arrays, half of which segregated along a horizontal axis and half of which segregated along a vertical axis. They were asked to respond by pressing the appropriate button (labeled $\mathrm{H}$ or V) as quickly as possible without making errors. The slides were presented for $200 \mathrm{msec}$ on the center of the screen. The observers were asked to maintain fixation on the center of the screen, where the stimulus slide would appear.

In a session, the observer was presented with four blocks of trials, two blocks for each of the two hue discriminability sets of stimuli (i.e., easy or hard). Within each block, 12 practice trials were followed by 100 experimental trials. The experimental trials consisted of five randomly ordered presentations of a set of 20 different slides. In the set of 20 stimulus slides there were 8 control arrays (4 control-hue, 4 control-brightness), 4 correlated arrays, and 8 orthogonal arrays (4 orthogonal-hue, 4 orthogonal-brightness). Half of the arrays were horizontally aligned and half vertically aligned for all subtypes. In addition, for control arrays, the dimension defining the fields was represented at both levels of 
the second dimension (e.g., for control-hue arrays both $\mathrm{H} 1 \mathrm{Bl}$ vs. $\mathrm{H} 2 \mathrm{~B} 1$ and $\mathrm{H} 1 \mathrm{~B} 2$ vs. H2B2 arrangements were used). For correlated arrays, both pairings of hue and brightness (i.e., H1B1 vs. H2B2 and H1B2 vs. H2B1) were included. Finally, for orthogonal arrays both positions of the two horizontal and two vertical field arrangements (e.g., $\mathrm{H} 1$ top $\mathrm{H} 2$ bottom, or vice versa, for the horizontal arrangement of orthogonal-hue arrays) were employed.

Type of stimulus array (control, correlated, orthogonal), as well as field orientation (horizontal, vertical), were randomized within a block. Hue discriminability (easy, hard) was balanced across blocks in a Latin square.

\section{Results}

Reaction time was measured from the onset of the stimulus array until the depression of the response button. These data were combined over the two blocks presented for each type of discriminability set (i.e., easy, hard), and then mean RTs for each subtype of stimulus array (i.e., control-hue, control-brightness, correlated, orthogonal-hue, orthogonal-brightness) were calculated.

Two analyses were performed on these mean RTs. In one, an analysis of variance assessed interference effects, which are indicated by longer mean RT in orthogonal than in control arrays. A 16 subjects $\times$ 2 hue discriminability (easy, hard) $\times 2$ type of stimulus array (control, orthogonal) $\times 2$ dimensions (hue, brightness) factorial design was employed in this analysis of variance. The Lindquist (1953) critical difference method was used in all a posteriori comparisons that resulted from this analysis. The level of significance achieved for these comparisons was $\mathrm{p}<.01$, unless otherwise noted. The second analysis assessed redundancy effects, which are revealed by a mean RT that is faster in correlated than in the faster of the two subtypes of control arrays (i.e., control-hue or control-brightness). In this analysis, a one-tailed $t$ test for matched groups was performed on each observer's fastest mean control RT, either control-hue or control-brightness, compared with his or her mean correlated RT.

\section{Interference Effects}

The data in Table 1 show the mean RTs for each dimension, type of stimulus array, and level of hue discriminability that correspond to the interference analysis of variance. The main effects that resulted from this analysis of variance are relatively unimportant and as expected. Overall mean RT for brightness arrays $(650 \mathrm{msec})$ was significantly faster than that for hue arrays $(772 \mathrm{msec})[\mathrm{F}(1,15)=127.51, \mathrm{p}<.01]$. An overall interference effect was manifested, with orthogonal arrays $(739 \mathrm{msec}$ ) being accompanied by longer mean RTs than control arrays $(683 \mathrm{msec})$ $[F(1,15)=37.41, p<.01]$. In addition, arrays in the hard discriminability $(763 \mathrm{msec})$ set of stimuli were responded to much more slowly on the average than those in the easy discriminability $(659 \mathrm{msec})$ set $[F(1,15)$ $=40.41, \mathrm{p}<.01]$.

The most important finding of this experiment was that the extent of interference due to irrelevant variation on the second dimension in orthogonal arrays depended on whether hue or brightness differences defined the fields. This effect was indicated by a significant hue discriminability $x$ type of stimulus array $\times$ dimension interaction $[F(1,15)=7.48, p<.01]$. Strong orthogonal interference occurred in both easy and hard arrays for hue arrays only. There was no interference evident for brightness arrays in either hue discriminability set. Further a posteriori tests of this interaction revealed that in both the easy $(p<.05)$ and hard sets, mean control RT was significantly faster for brightness than for hue arrays.

In general, the manipulation of hue discriminability tended to enhance obtained effects more in hard than in easy sets of stimuli. Thus, the interference, revealed by a comparison of RT for control and orthogonal arrays, was much greater for the hard (724 vs. $802 \mathrm{msec})$ than for the easy (642 vs. $676 \mathrm{msec}$ ) discriminability set of stimuli $[F(1,15)=13.67, p<.01]$. Likewise, when levels of hue were hard rather than easy to discriminate, mean RT was sharply increased for hue arrays (856 vs. $688 \mathrm{msec}$ ) and less sharply increased for brightness arrays (670 vs. $630 \mathrm{msec}$ ), as revealed by a significant hue discriminability $\times$ dimension interaction $[F(1,15)=80.48, p<.01]$. This increase in mean RT for brightness arrays occurred across stimulus sets, even though only hue discriminability was varied.

\section{Redundancy Effects}

The results of $t$ tests revealed that the mean $R T$ was equivalent for correlated and the faster of the

Table 1

Mean Reaction Time (Milliseconds) for Correct Segregation Responses and Mean Proportion of Errors (in Parentheses) in the Interference Analysis of Experiment 1

\begin{tabular}{|c|c|c|c|c|}
\hline \multirow{2}{*}{$\begin{array}{c}\text { Relevant } \\
\text { Dimension }\end{array}$} & \multirow{2}{*}{$\begin{array}{c}\text { Hue } \\
\text { Discriminability }\end{array}$} & \multicolumn{3}{|c|}{ Type of Stimulus Array } \\
\hline & & Control & Orthogonal & Combined \\
\hline Hue & $\begin{array}{l}\text { Easy } \\
\text { Hard } \\
\text { Combined }\end{array}$ & $\begin{array}{l}659(.03) \\
785(.05) \\
722(.04)\end{array}$ & $\begin{array}{l}717(.06) \\
926(.08) \\
821(.07)\end{array}$ & $\begin{array}{l}688(.05) \\
856(.07) \\
772(.06)\end{array}$ \\
\hline Brightness & $\begin{array}{l}\text { Easy } \\
\text { Hard } \\
\text { Combined }\end{array}$ & $\begin{array}{l}624(.02) \\
662(.02) \\
643(.02)\end{array}$ & $\begin{array}{l}635(.01) \\
678(.03) \\
657(.02)\end{array}$ & $\begin{array}{l}630(.02) \\
670(.03) \\
650(.02)\end{array}$ \\
\hline
\end{tabular}


two subtypes of control arrays. This was true for both easy ( $623 \mathrm{msec}$ for fastest control vs. $630 \mathrm{msec}$ for correlated arrays) and hard (662 msec for fastest control vs. $675 \mathrm{msec}$ for correlated arrays) sets of stimuli. The faster subtype of control array was brightness for $13 / 16$ observers (one-tailed $p<.002$ ) in the easy set and for all 16 observers in the hard set.

\section{Errors}

The mean proportion of errors for each dimension, type of stimulus array, and hue discriminability level are given in parentheses in Table 1 . The pattern found is similar to that of the RT data, and analysis of errors would not significantly add to our understanding of those results. The highest mean proportion of errors is found for hue arrays in the hard hue discriminability set of stimuli.

\section{Discussion}

A strong indication for the integrality of hue and brightness dimensions is given by symmetric interference when dimensions are varied orthogonally and by redundancy gains when dimensions are correlated (Garner \& Felfoldy, 1970). The present data do not show this characteristic pattern. There was no evidence that correlation of dimensions enhanced performance beyond that found for the faster of the two subtypes of control arrays. In addition, the findings indicate that irrelevant variation of brightness (i.e., in orthogonal-hue arrays) interferes far more with segregation judgments based on hue differences than the converse (see Table 1). Hue variation did not significantly interfere with brightness judgments. Further, the manipulation of hue discriminability resulted in significantly longer mean RTs for both hue and brightness arrays, even though only hue discriminability was varied in this manipulation. One explanation for the anomalous finding is that the grouping together of brightness with hue arrays in blocks according to hue discriminability may have produced a general slowing of response to all arrays in the hard discriminability blocks. This possibility was assessed in Experiment 2 , in which all stimulus arrays were randomly intermixed in an unblocked design.

\section{EXPERIMENT 2}

\section{Method}

\section{Experimental Conditions}

All aspects of this experiment were identical to those of Experiment 1 with the single exception of the conditions of blocking. In Experiment 1, observers were presented with four blocks of 100 trials, two blocks for each hue discriminability set. One possible solution to the problem of grouping hard stimulus arrays together is to block stimulus arrays according to dimension (i.e., hue or brightness). It was felt that this form of blocking could have produced other potential problems; therefore, in Experiment 2, an unblocked procedure was adopted. An unblocked procedure should help to ensure that the observer responds, as instructed, to the overall orientation of fields in the whole stimulus array.

In Experiment 2, stimulus arrays from the two hue discriminability sets were mixed. Within a session, the observer was presented with 400 experimental trials. The experimental trials consisted of 10 randomly ordered presentations of each of the 40 stimulus slides. The stimulus slides, identical to those of Experiment 1 , included 16 control arrays ( 4 control-hue easy, 4 controlhue hard, 4 control-brightness easy, 4 control-brightness hard), 8 correlated arrays ( 4 correlated easy, 4 correlated hard), and 16 orthogonal arrays (4 orthogonal-hue easy, 4 orthogonal-hue hard, 4 orthogonal-brightness easy, 4 orthogonal-brightness hard).

In order to preserve the general procedural quality of Experiment 1 , the experimenter stopped for a break after each 100 trials in Experiment 2, and began each new "block" with 12 practice trials.

\section{Subjects}

Sixteen new Yale undergraduates served in this experiment. All had normal, or corrected, vision. Each observer participated in one 1-h session and was paid for participation.

\section{Results}

The mean RTs for each subtype of array in this experiment were calculated and then analyzed as in Experiment 1.

\section{Interference Effects}

The data in Table 2 show mean RTs for each dimension, type of stimulus array, and level of hue discriminability that were obtained in the interference analysis of Experiment 2. As in Experiment 1, all main effects and interactions were significant in the overall analysis of variance. The main effects were identical to those of Experiment 1. Overall mean RT for brightness arrays (586 msec) was significantly faster than that for hue arrays $(718 \mathrm{msec})[F(1,15)=119.11$, $\mathrm{p}<.01]$. An overall interference effect was evident, with orthogonal arrays $(672 \mathrm{msec})$ showing longer mean RTs than control arrays $(632 \mathrm{msec})[\mathrm{F}(1,15)=$ $24.77, p<.01$ ]. As in Experiment 1, arrays for which hues were hard to discriminate $(684 \mathrm{msec})$ were responded to more slowly on the average than those for which hue differences were easy to discriminate $(620 \mathrm{msec})[\mathrm{F}(1,15)=51.55, \mathrm{p}<.01]$.

The salient finding of this experiment was a replication of the strong orthogonal interference for hue arrays at both levels of hue discriminability reported in Experiment 1. Also, in accord with Experiment 1, there was no interference evident for brightness arrays at either level of hue discriminability. This pattern of asymmetry was revealed by a posteriori tests of the hue discriminability $\times$ type of stimulus array $x$ dimension interaction $[F(1,15)=8.41, p<.01]$. As in Experiment 1, mean control RT was significantly faster for brightness than for hue arrays in both easy ( 580 vs. $636 \mathrm{msec})$ and hard (585 vs. 726 msec) sets.

In contrast to Experiment 1, only hue arrays were affected by the hue discriminability manipulation. When levels of hue were hard rather than easy to dis- 
criminate, mean RT significantly increased for hue arrays (780 vs. $657 \mathrm{msec}$ ) and remained constant for brightness arrays ( 588 vs. $583 \mathrm{msec})[\mathrm{F}(1,15)=70.72$, $\mathrm{p}<.05]$. The manipulation of hue discriminability tended, as in Experiment 1, to enhance the obtained interference effects. Although the first-order interaction of hue discriminability $\times$ type of stimulus array is significant $[F(1,15)=17.61, p<.01]$, it is clear from Table 1 that the effect was confined to hue arrays. Thus, the interference, reflected by longer mean RT for orthogonal-hue than for control-hue arrays, was much greater for hard (833 vs. $726 \mathrm{msec})$ than for easy (678 vs. $636 \mathrm{msec}$ ) discriminability sets of stimuli.

\section{Redundancy Effects}

The results of the $t$ tests revealed that mean $R T$ was equivalent for correlated and the faster of the two subtypes of control arrays. This effect replicates Experiment 1 and was true for both easy $(578 \mathrm{msec}$ for fastest control vs. $589 \mathrm{msec}$ for correlated arrays) and hard (585 msec for fastest control vs. $577 \mathrm{msec}$ for correlated arrays) stimulus sets. The faster subtype of control array was brightness for 15 of $16 \mathrm{ob}$ servers (one-tailed $p<.001$ ) in the easy set, and for all 16 observers in the hard set.

\section{Errors}

The mean proportion of errors for each dimension, type of stimulus array, and hue discriminability level are provided in parentheses in Table 2. As in Experiment 1, the pattern of errors was similar to that of RT data, with the highest proportion of errors being found for hue arrays in the hard discriminability set.

\section{Discussion}

The findings of Experiment 2 clarify the pattern of asymmetric interference found in Experiment 1 . As expected, removal of blocking according to hue discriminability eliminated the anomalous result of longer mean RTs for brightness arrays in the hard than in the easy discriminability set. Thus, there was a general slowing of response for Experiment 1 when arrays were blocked in the hard stimulus set. The data from Experiments 1 and 2 do not have the characteristic integral pattern of symmetric orthogonal interference and redundancy gains for correlated dimensions. There was no redundancy gain in either experiment. In addition, the pattern of interference was an asymmetric one. For both experiments, the irrelevant variation of brightness in orthogonal-hue arrays produced strong interference, in contrast to the lack of interference for orthogonal-brightness arrays.

Previous research has indicated that the relative discriminability of dimensions in a stimulus set has a significant impact on the pattern of dimensional interaction effects. Garner and Felfoldy (1970, Experiment 5) varied the discriminability of brightness and saturation stimuli in a speeded sorting task and found that a highly discriminable set of stimuli was accompanied by less interference than a set containing less discriminable stimuli. The finding, in the present experiments, of main effects of hue discriminability and significant interactions with both dimension and type of stimulus array factors attests to the potency of this variable in a preattentive field-segregation task. In line with the Garner and Felfoldy results, we find that interference for hue arrays in the hard discriminability set (differences of $141 \mathrm{msec}$ in Experiment 1 and $107 \mathrm{msec}$ in Experiment 2 between the control and orthogonal arrays) is over twice that found in the easy discriminability set (a difference of $58 \mathrm{msec}$ in Experiment 1 and $42 \mathrm{msec}$ in Experiment 2 between the control and orthogonal arrays).

Lack of interference due to the irrelevant variation of hue features in orthogonal-brightness arrays may be due to the operation of a dimension discriminability mechanism. Specifically, it is possible that the levels of brightness chosen for the construction of the stimulus arrays used in Experiments 1 and 2 (i.e., 3 and 5 in the Munsell system) were so easily discriminated that a ceiling effect obscured interference due to hue variation in orthogonal arrays. Furthermore, with stimuli for which overall similarity relations are important (i.e., integral stimuli), a redundancy gain is maximized when the discriminabilities between levels on component dimensions is equivalent (Garner,

Table 2

Mean Reaction Time (Milliseconds) for Correct Segregation Responses and Mean Proportion Errors (in Parentheses) in the Interference Analysis of Experiment 2

\begin{tabular}{|c|c|c|c|c|}
\hline \multirow{2}{*}{$\begin{array}{c}\text { Relevant } \\
\text { Dimension }\end{array}$} & \multirow{2}{*}{$\begin{array}{c}\text { Hue } \\
\text { Discriminability }\end{array}$} & \multicolumn{3}{|c|}{ Type of Stimulus Array } \\
\hline & & Control & Orthogonal & Combined \\
\hline Hue & $\begin{array}{l}\text { Easy } \\
\text { Hard } \\
\text { Combined }\end{array}$ & $\begin{array}{l}636(.02) \\
726(.08) \\
681(.05)\end{array}$ & $\begin{array}{l}678(.02) \\
833(.08) \\
756(.05)\end{array}$ & $\begin{array}{l}657(.02) \\
780(.08) \\
718(.05)\end{array}$ \\
\hline Brightness & $\begin{array}{l}\text { Easy } \\
\text { Hard } \\
\text { Combined }\end{array}$ & $\begin{array}{l}580(.02) \\
585(.02) \\
583(.02)\end{array}$ & $\begin{array}{l}585(.01) \\
591(.02) \\
588(.02)\end{array}$ & $\begin{array}{l}583(.02) \\
588(.02) \\
586(.02)\end{array}$ \\
\hline
\end{tabular}


1974). In terms of the present situation, this means that a redundancy gain is most likely to occur for a stimulus set with equivalent control-hue and controlbrightness mean RTs, a condition that was not realized in the present experiments. In the easy set, 13 of 16 observers in Experiment 1 (one-tailed $p<.002$ ) and 15 of 16 observers in Experiment 2 (one-tailed $p<.001$ ) showed faster mean control RT for brightness than for hue arrays. In the hard set, all 16 observers showed faster mean control RT for brightness arrays in both experiments.

Since the pattern of interference found in this experiment was directly influenced by relative discriminability of dimensions, it was decided to assess preattentive dimensional interaction at other levels of hue and brightness discriminability. In Experiment 3, two new sets of hue and brightness stimuli were constructed. In the new stimuli, brightness discriminability was considerably reduced, and hue discriminability was enhanced. It was expected that these new stimulus conditions would result in symmetric interference of hue variation on brightness arrays and of brightness variation on hue arrays.

\section{EXPERIMENT 3}

\section{Method}

\section{Experimental Conditions}

In this experiment, all aspects of array construction and procedure were identical to those of Experiment 1, with the exception of the particular levels on the hue and brightness dimensions that were used in making the stimuli. For the easy discriminability set of stimuli in this experiment, levels of hue were separated by five Munsell steps $(\mathrm{H1}=7.5 \mathrm{R}, \mathrm{H} 2=5 \mathrm{RP})$, and levels of brightness, by one step $(\mathrm{B} 1=3, \mathrm{~B} 2=4)$. This is in contrast to the easy discriminability set of Experiment 1, for which hue levels were separated by four and brightness by two Munsell steps. In Experiment 3, the hard discriminability set of stimuli was constructed from levels of hue that were separated by three Munsell steps $(H 1=7.5 R$, $\mathrm{H} 2=10 \mathrm{RP}$ ) and from levels of brightness that differed by one step $(B 1=3, B 2=4)$. Again, this contrasts with the hard discriminability set from Experiment 1 , for which both hue and brightness levels differed by two steps. As in Experiment 1, all elements in both easy and hard sets had a constant chroma level of 8 .

\section{Subjects}

Sixteen new Yale undergraduates served in this experiment. All had normal, or corrected, vision. Each observer participated in one 1-h experimental session and was paid for his or her participation.

\section{Results}

As in Experiment 1, the mean RTs for each subtype of array, averaged over two blocks, were subjected to two analyses. Interference effects were assessed by a 16 subjects $\times 2$ hue discriminability (easy, hard) $\times 2$ type of stimulus array (control, orthogonal) $\times 2$ dimensions (hue, brightness) factorial analysis of variance. The Lindquist (1953) critical difference was employed in a posteriori tests that were warranted by this analysis. All tests were significant at a level of $p<.01$. Redundancy effects were assessed with one-tailed $t$ tests, which compared each observer's fastest mean control RT with his or her mean correlated RT.

\section{Interference Effects}

The data in Table 3 show mean RTs for each dimension, type of stimulus array, and level of hue discriminability that were obtained in the interference analysis of Experiment 3. As in Experiment 1, overall mean RT for brightness arrays $(650 \mathrm{msec})$ was significantly faster than that for hue arrays $(725 \mathrm{msec})$ $[F(1,15)=9.35, \mathrm{p}<.01]$. Again, in line with Experiment 1 , overall mean $\mathrm{RT}$ was significantly longer for orthogonal $(712 \mathrm{msec})$ than for control $(663 \mathrm{msec})$ arrays. This interference effect was revealed by a significant main effect of type of stimulus array $[F(1,15)$ $=11.35, \mathrm{p}<.01]$. Arrays in the hard discriminability set of stimuli $(714 \mathrm{msec})$ were responded to much more slowly on the average than those in the easy set $(661 \mathrm{msec})[\mathrm{F}(1,15)=11.16, \mathrm{p}<.01]$. In contrast to Experiment 1 , the manipulation of hue discriminability slowed mean RT more in hard than in easy sets for hue (783 vs. $666 \mathrm{msec}$ ), but not for brightness (644 vs. $656 \mathrm{msec})$ arrays $[F(1,15)=22.80$, p $<.01]$.

The important finding of Experiment 3 was a symmetric interference effect that was obtained in the easy discriminability set of stimuli $[F(1,15)=15.73$, $p<.01$ ]. That is, for the easy set, irrelevant variation of brightness resulted in a mean RT that was longer in the orthogonal-hue $(682 \mathrm{msec})$ array than in the

Table 3

Mean Reaction Time (Milliseconds) for Correct Segregation Responses and Mean Proportion of Errors (in Parentheses) in the Interference Analysis of Experiment 3

\begin{tabular}{|c|c|c|c|c|}
\hline \multirow{2}{*}{$\begin{array}{c}\text { Relevant } \\
\text { Dimension }\end{array}$} & \multirow{2}{*}{$\begin{array}{c}\text { Hue } \\
\text { Discriminability }\end{array}$} & \multicolumn{3}{|c|}{ Type of Stimulus Array } \\
\hline & & Control & Orthogonal & Combined \\
\hline Hue & $\begin{array}{l}\text { Easy } \\
\text { Hard } \\
\text { Combined }\end{array}$ & $\begin{array}{l}650(.02) \\
734(.06) \\
692(.04)\end{array}$ & $\begin{array}{l}682(.04) \\
832(.11) \\
757(.08)\end{array}$ & $\begin{array}{l}666(.03) \\
783(.09) \\
725(.06)\end{array}$ \\
\hline Brightness & $\begin{array}{l}\text { Easy } \\
\text { Hard } \\
\text { Combined }\end{array}$ & $\begin{array}{l}628(.03) \\
640(.01) \\
634(.02)\end{array}$ & $\begin{array}{l}684(.02) \\
649(.02) \\
666(.02)\end{array}$ & $\begin{array}{l}656(.03) \\
644(.02) \\
650(.02) \\
\end{array}$ \\
\hline
\end{tabular}


control-hue $(650 \mathrm{msec})$ array, and irrelevant variation of hue produced a mean RT that was longer in the orthogonal-brightness $(684 \mathrm{msec})$ array than in the control-brightness $(628 \mathrm{msec})$ array. In the easy set, mean control RTs were not significantly different for hue and brightness arrays (650 vs. $628 \mathrm{msec}$ ). In the hard arrays, interference was evident only for hue arrays, where mean RT was $832 \mathrm{msec}$ for orthogonalhue and $734 \mathrm{msec}$ for control-hue arrays. For the hard set, mean RT for control-hue was significantly longer than that for control-brightness (734 vs. $640 \mathrm{msec}$ ).

In contrast to Experiment 1, mean RT for orthogonal-brightness arrays was found to be significantly faster in the hard than in the easy stimulus set (649 vs. $684 \mathrm{msec}$ ). This is expected if we assume that as hue becomes harder to discriminate it should interfere less with judgments based on brightness. Both subtypes of hue arrays showed longer mean RT in hard than in easy sets (734 vs. $650 \mathrm{msec}$ for controlhue, 832 vs. $682 \mathrm{msec}$ for orthogonal-hue), whereas mean RT for control-brightness arrays did not change significantly (640 vs. $628 \mathrm{msec}$ ).

\section{Redundancy Effects}

The results of $t$ tests showed that mean RT for correlated arrays in Experiment 3 was equivalent to the faster of the two control arrays. This was true for both easy $(625 \mathrm{msec}$ for faster control vs. $617 \mathrm{msec}$ for correlated), and hard $(640 \mathrm{msec}$ for faster control vs. $640 \mathrm{msec}$ for correlated) sets of stimuli. The faster subtype of control array was control-brightness for 11 of 16 observers (one-tailed $p<.04$ ) with the easy set, and for all 16 observers with the hard set. These findings are consistent with those of Experiment 1 .

\section{Errors}

The mean proportion of errors for Experiment 3 are given in parentheses in Table 3. As in the previous two experiments, the pattern for errors reflected that of the RT data, with the highest mean proportion of errors found in hue arrays in the hard discriminability set.

\section{Discussion}

The results of Experiment 3 confirmed that relative discriminability of component dimensions has a significant impact on degree of dimensional interaction. In this experiment, the discriminability of hue was enhanced and the discriminability of brightness was decreased, relative to the levels used in Experiment 1. With the new stimuli, symmetric interference of hue on brightness, and brightness on hue, was obtained for the easy set of stimuli. This is in contrast to the asymmetric interference obtained in Experiments 1 and 2 , but is expected on the basis of the change in relative discriminability of dimensions for these stimulus arrays. In Experiment 3, as in the first two experiments, correlation of dimensions did not result in the usual performance gains that characterize integral stimuli. Lack of a redundancy gain in all experiments suggests that preattentive field segregation is not enhanced by a redundant combination of dimensional information.

\section{GENERAL DISCUSSION}

The goal of these experiments was to assess the extent of dimensional interaction for hue and brightness during preattentive processing using a novel technique. The task we employed, a reaction time field segregation task, was found to be highly sensitive to interference effects. The findings of Experiments 1 and 2 showed, as expected, interference for segregation judgments based on hue when brightness levels varied orthogonally in the array. However, the converse result of interference in segregation judgments based on brightness when hue varied was not manifested. The results of Experiment 3 indicated that the asymmetric pattern found in the first two experiments may have been due to the high discriminability between brightness levels used in the construction of arrays for those experiments. When relative discriminabilities were altered in Experiment 3, such that brightness levels were less discriminable but hue levels more so, symmetric interference of hue on brightness and brightness on hue was evident, but only for the easy stimulus set.

Beck (1982, Beck et al., 1983) and Treisman (1982, in press; Treisman \& Gelade, 1980) argue that simple properties of the stimulus (i.e., color, shape, size, orientation, etc.) mediate field segregation in complex visual arrays. Our results corroborate and extend these findings. In our arrays, fields are defined by a difference either in hue or brightness, or by a redundant combination of levels on these dimensions. Evidence that hue as well as brightness differences successfully established field segregation in these experiments is gained by the finding of very few errors (mean proportion of errors in Experiment 1 $=0.04$, in Experiment $2=0.03$, in Experiment $3=$ 0.04).

Moreover, the present results argue strongly for the conclusion that although these dimensions can be used to produce preattentive field segregation, they are not immune from interference due to the variation of other properties present in the stimulus array. In Beck's (1982) terms, the irrelevant variation of hue or brightness dimensions across the orthogonal arrays serves to decrease element similarity within regions, thereby interfering with the segregation response. In addition, the variation of dimensions across an entire array could produce a grouping of elements that is contrary to the one intended by the experimenter. 
For example, in the orthogonal-hue arrays of the present experiments, observers sometimes reported that all the "bright" elements stood out, and the array appeared as a checkerboard pattern rather than as horizontally or vertically aligned regions. In this way, the properties of elements across experimenter-defined regions may increase in similarity. According to Beck (1982), increasing similarity of simple property differences across regions is another way of interfering with field segregation.

Unlike the typical result for attentional tasks, field segregation does not appear to be enhanced by redundant combination of levels on component dimensions. According to Beck's (1982) model, redundant combination of dimensions should serve to decrease element similarity between groups and increase similarity within groups, and hence to improve segregation. The particular reason for the lack of a redundancy gain with these stimuli is not clear. There are a number of possible explanations. For one, brightness may be a more effective mediator of field segregation than hue. Furthermore, redundancy gains are typically found in attentional experiments when observers are presented with correlated arrays blocked separately from control arrays. It is possible that concentrated exposure to a single subtype of array may increase the likelihood of redundancy gains. However, the most probable explanation of this lack is that redundancy gains are not typically found unless component dimensions are equally discriminable. Equivalent discriminability was not evident for any of the present sets of stimuli. The only exception to this was that, in Experiment 3, control-hue and control-brightness arrays did not significantly differ in the easy discriminability set $(650 \mathrm{vs} .628 \mathrm{msec})$. However, even for this set of stimuli 11 of 16 observers showed faster mean RT for control-brightness than for control-hue arrays. Thus, in gerieral, brightness arrays were more discriminable than hue arrays. The viability of these alternatives should be explored at other levels of dimensional discriminability.

\section{REFERENCES}

Beck, J. (1966). Effect of orientation and of shape similarity on perceptual grouping. Perception \& Psychophysics, 1, 300-302.

BEck, J. (1967). Perceptual grouping produced by line figures. Perception \& Psychophysics, 2, 491-495.

BECK, J. (1972). Similarity grouping and peripheral discriminability under uncertainty. American Journal of Psychology, 85, $1-19$.

BEcK, J. (1982). Textural segmentation. In J. Beck (Ed.), Organization and representation in perception. Hillsdale, $\mathrm{NJ}$ : Erlbaum.

Beck, J., Prazdny, K., \& Rosenfeld, A. (1983). A theory of textural segmentation. In J. Beck, B. Hope, \& A. Rosenfeld (Eds.), Human and machine vision. New York: Academic Press. Burns, B. B., She Pp, B. E., McDonough, D., \& Erlich, W. (1978). The relation between stimulus analyzability and perceived dimensional structure. In G. H. Bower (Ed.), The psychology of learning and motivation: Advances in research and theory (Vol. 12). New York: Academic Press.

Garner, W. R. (1970). The stimulus in information processing. American Psychologist, 25, 350-358.

GARNER, W. R. (1974). The processing of information and structure. Potomac, MD: Erlbaum.

GaRnER, W. R. (1976). Interaction of stimulus dimensions in concept and choice processes. Cognitive Psychology, 8, 98-123.

Garner, W. R. (1978). Aspects of a stimulus: Features, dimensions, and configurations. In E. Rosch \& B. B. Lloyd (Eds.), Cognition and categorization. Hillsdale, NJ: Erlbaum.

GarNe R, W. R. (1983). Asymmetric interactions of stimulus dimensions in perceptual information processing. In T. J. Tighe \& B. E. Shepp (Eds.), Interactions: Perception, cognition, and development: A Second Dartmouth Multi-Perspective Conference. Hillsdale, $\mathrm{NJ}$ : Erlbaum.

Garner, W. R., \& Felfoldy, G. L. (1970). Integrality of stimulus dimensions in various types of information processing. Cognitive Psychology, 1, 225-241.

GotTwald, R. L., \& Garner, W. R. (1975). Filtering and condensation tasks with integral and separable stimuli. Perception \& Psychophysics, 18, 26-28.

Handel, S., \& ImaI, S. (1972). The free classification of analyzable and unanalyzable stimuli. Perception \& Psychophysics, 12, 108-116.

Hyman, R., \& Well, A. (1967). Judgments of similarity and spatial models. Perception \& Psychophysics, 2, 233-248.

Kahneman, D. (1973). Attention and effort. Englewood Cliffs, NJ: Prentice-Hall.

LINDQUIST, E. F. (1953). Design and analysis of experiments in psychology and education. New York: Houghton Mifflin.

Neisser, U. (1967). Cognitive psychology. New York: AppeltonCentury-Crofts.

She PP, B. E. (1983). The analyzability of multidimensional stimuli: Some constraints on perceived structure and attention. In T. J. Tighe \& B. E. Shepp (Eds.), Interactions: Perception, cognition, and development: A Second Dartmouth MultiPerspective Conference. Hillsdale, NJ: Erlbaum.

She Pp, B. E., \& Swartz, K. B. (1976). Selective attention and the processing of integral and nonintegral dimensions: A developmental study. Journal of Experimental Child Psychology, 22, 73-85.

Torgerson, W. S. (1958). Theory and methods of scaling. New York: Wiley.

Treisman, A. M. (1979). The psychological reality of levels of processing. In L. S. Cermak \& F. I. M. Craik (Eds.), Levels of processing in human memory. Hillsdale, $\mathrm{NJ}$ : Erlbaum.

Treisman, A. M. (1982). Perceptual grouping and attention in visual search for features and for objects. Journal of Experimental Psychology: Human Perception and Performance, 8, 194-214.

Treisman, A. (in press). Properties, parts, and objects. In K. Boff, L. Kaufman, \& J. Thomas (Eds.), Handbook of perception and human performance. New York: Wiley.

Treisman, A., \& Gelade, G. (1980). A feature-integration theory of attention. Cognitive Psychology, 12, 97-136.

(Manuscript received December 2, 1983; revision accepted for publication June 4,1984 .) 